\title{
Nutrition-related patient safety incidents
}

\author{
B. Holmes ${ }^{1}$, A. Spiro ${ }^{1}$, J. E. Thomas ${ }^{1}$, K. Whelan ${ }^{1}$, C. E. Weekes ${ }^{2}$, C. Baldwin ${ }^{1}$ and P. W. Emery ${ }^{1}$ \\ ${ }^{1}$ Nutritional Sciences Division, King's College London, London SE1 9NH, UK and ${ }^{2}$ Department of Nutrition and Dietetics, \\ Guys and St Thomas' NHS Foundation Trust, London SE1 7EH, UK
}

Nutrition is an essential part of patient care and inappropriate or absent care can be a cause of safety problems in some patients. The National Patient Safety Agency (NPSA) maintains a register of all safety incidents reported in the UK through the National Reporting and Learning Service (NRLS). The aim of this project was to analyse and classify the most commonly reported nutrition-related patient safety incidents and to identify key common themes that emerge from the data.

The text of all incidents recorded on the NRLS in 2006 and 2007 (1.6 million reports) was searched by the NPSA using a series of nutrition-related keywords to produce a subset of 52675 potentially nutrition-related incidents. A random sample of 4992 of these incident reports was reviewed in detail. Incidents were classified as nutrition related if they met the following definition: An incident in which the provision of nutrition (or nutritional services) either caused harm or had the potential to cause harm to an individual.

After excluding duplicate reports, 1433 incidents ( $29 \%$ of the total) met the definition of nutrition related and were classified into 13 broad categories (see Table).

\begin{tabular}{lcc}
\hline Category & Number of incidents & Percent of total \\
\hline Provision of nutrition via artificial feeding & 328 & 23 \\
Nil by mouth/fasting & 326 & 23 \\
Provision of nutrition via oral feeding & 247 & 17 \\
Nutritional assessment or support & 158 & 11 \\
Discharge related/community assistance in relation to feeding & 108 & 8 \\
Food hygiene and food safety & 82 & 6 \\
Diabetes and blood glucose levels & 64 & 5 \\
Consequences of malnutrition & 55 & 4 \\
Fluid management & 47 & 3 \\
Falls/slips/trips with nutritional involvement & 28 & 2 \\
Patient refusals of food or drink & 17 & 1 \\
Allergy & 9 & 1 \\
Other or insufficient information available & 15 & 1 \\
\hline
\end{tabular}

The greatest number of incidents involved artificial feeding. Half of these related to delayed or incorrect placement of the tube or pumps that were faulty or turned off. The rest related to the feeds, either incorrect type, insufficient amount or wrong feeding rate or regimen. Incidents relating to patients being nil by mouth were equally split between failure to implement this or it being prolonged or inappropriate. Oral feeding incidents predominantly involved inappropriate type or texture of food, followed by inadequate amounts of food, late or non-arrival of meals and a small number of incidents where a lack of adequate feeding assistance was reported. The inadequate nutritional assessment and support category included delays in referring patients to dietitians or speech and language therapists. Incidents related to consequences of malnutrition were largely concerned with the development of pressure sores, where malnutrition was highlighted as a major cause. The relatively small number of incidents classified in this category (4\%) may reflect the recognition that malnutrition may be only one of several contributing causes, and/or a lack of recognition of the potential contribution of malnutrition to the cases. In fact, malnutrition is itself a patient safety issue.

Common themes that emerged from these nutrition-related patient safety incidents included poor communication between staff and departments, inadequate documentation concerning food and fluid requirements, inadequate staffing levels and insufficiently trained or skilled staff to provide satisfactory nutritional care. 